Methods All paediatricians involved in aetiological assessment were identified from the national audiology lead. A questionnaire was developed using the BAAP standards and was sent by post with email reminders.

Results were collated on excel.

Results 38 consultants were identified of whom 37 responded. 33 paediatricians reported that they assessed children with PCHI in clinic. Only 6/33 had received any specific training in the assessment of PCHI infants. This work was not included in any job plan and no additional posts had been developed. Only 1 paediatrician reported participating in a regional support network, but the vast majority (92.9\%) surveyed expressed an interest in developing a regional network. $5 / 33(15 \%)$ saw the infants in a dedicated hearing clinic.

Waiting time for assessment was beyond the recommended timeframe in all cases, (range 2-52 weeks, average of 14.4 weeks). Only $8 / 29(27 \%)$ assess by 6 weeks of age, the time limit for treatment of congenital CMV infection. Access to MRI is limited, only $12(28.6 \%)$ reported having access to MRI within 12 weeks of age thus the majority of MRI require sedation. $77 \%$ routinely refer all children with $\mathrm{PCHI}$ to ophthalmology.

Conclusion The above survey highlights significant deficits in the paediatric component of the UNHS. Identified gaps include; inadequate appropriately trained staff and lack of timely access to necessary investigations and tertiary services.

A national multi-disciplinary working group has been developed to address the deficits and ensure an improvement in service provision.

\section{G465 A REGIONAL QUALITY IMPROVEMENT PROJECT FOR CONGENITAL CYTOMEGALOVIRUS ASSOCIATED SENSORINEURAL HEARING LOSS}

\begin{abstract}
${ }^{1,2} \mathrm{~T}$ Holland Brown, ${ }^{3} \mathrm{FE}$ Walston, ${ }^{4} \mathrm{KEM}$ McDevitt. 'Department of Community Paediatrics and Child Health, Cambridgeshire Community Services, Cambridge, UK; ${ }^{2}$ Department of Research and Development, Cambridgeshire Community Services, Cambridge, UK; ${ }^{3}$ Department of Neonatology, Norfolk and Norwich University Hospitals, NHS Foundation Trust, Norfolk, UK; ${ }^{4}$ Department of General Paediatrics, North West Anglia NHS Foundation Trust, Peterborough, UK
\end{abstract}

\subsection{6/archdischild-2018-rcpch.453}

Aims Treating congenital Cytomegalovirus (cCMV) associated sensorineural hearing loss with ganciclovir has been shown to improve hearing outcomes. ${ }^{1,2}$ This project was set up to introduce a region-wide approach to testing, diagnosing and offering treatment to infants with cCMV associated sensorineural hearing loss before 28 days of life.

Methods A region wide study day on cCMV associated hearing loss was organised to which all paediatricians, hearing screeners, audiologists, ENT surgeons and virologists at the region's hospitals were invited. Speakers included international researchers in the area of cCMV, parents of children with hearing loss and clinicians who had set up a cCMV pathway elsewhere. Following this, a subgroup of interested clinicians, laboratory scientists and screeners was set up to investigate the potential for a region wide approach. The group met three times and was truly multi-disciplinary. Between meetings different multi-disciplinary groups worked on elements of the pathway. Beyond those involved in the subgroup, other clinicians and specialists such as ophthalmologists, radiologists and pharmacists contributed to the final pathway.
Results a) audits confirmed that, prior to implementation, cCMV was diagnosed too late, if at all, for ganciclovir treatment b) the regional virology services introduced CMV PCR testing on salivary samples with appropriate validation c) hearing screeners confirmed the acceptability of screeners taking salivary samples d) a generic regional pathway was introduced allowing for local adaptation and implementation

Conclusions Truly multi-disciplinary, cross-region working allows for rapid design and implementation of a project in a consistent and timely manner. Although clinician led, the regional hearing screening teams were instrumental in getting the project off the ground.

\section{REFERENCES}

1. Griffiths Paul D. The treatment of cytomegalovirus infection. Journal of Antimicrobial Chemotherapy 2002;49(2):243-253.

2. Shah $T$, Luck $S$, Sharland $M$, et al. Fiftenn-minute consultation: Diagnosis and management of congenital CMV. Arch Dis Child Educ Pract Ed 2016;101:232235.

\section{George Still Forum (National Paediatric ADHD Network)}

\section{G466 THE NEED AND RISE OF ADHD SERVICE IN A DGH SETTING}

S Nelapatla, V Pulla. Paediatrics, Northern Lincolnshire and Goole NHS Foundation Trust, Scunthorpe, UK

\subsection{6/archdischild-2018-rcpch.454}

Aim There are no national or regional models of care...in CAMHS or Paediatrics.

To share our experience of our model of care in setting up and running ADHD service (fully compliiant with NICE guidelines) for nearly 20 years, actively managing about 200 children per year in a borough with child population of 40000 .

We will be presenting care pathway, customised templates, assessments, monitoring, resources, stake holder involvement, specialist nurse role, specialist and speciality trainee involvement and transtion arrangements to adult care.

Conclusion Mental Health - The new epidemic needs novel ways and models of care.

Our model demonstrates collaborative working, efficient use of limited NHS resources in ever increasing demand on Paediatric and CAMHS services.

\section{G467 MANAGEMENT OF SLEEP DIFFICULTIES AMONG A COHORT OF CHILDREN WITH ADHD IN A SCOTTISH LOCAL AUTHORITY}

MO Ogundele. Community Paediatrics Unit, NHS Fife, Glenwood Health Centre, Glenrothes, UK

\subsection{6/archdischild-2018-rcpch.455}

An estimated 25\%-50\% of children and adolescents with ADHD are known to experience problems with sleep, which is approximately five-fold that of healthy controls. ADHDrelated sleep problems include high rates of daytime sleepiness, increased risk of sleep disordered breathing $(50 \%$ vs $22 \%$ in controls), restless legs syndrome and periodic leg movement disorder. Sleep deprivation may mimic and 
exacerbate many symptoms of ADHD, impair scholastic performance and cause memory problems.

Aims We aimed to analyse the prevalence of ADHD and sleep problems among children and young people seen within local Community Child Health clinics of a Scottish NHS Trust over a 12 month period.

Methods A retrospective review of all patients seen in the outpatient clinics between June 2016 and May 2017 in two different clinics within NHS Fife was carried out. ADHD was diagnosed using validated Swanson, Nolan, and Pelham -IV Questionnaire (SNAP-IV). Sleep problems were diagnosed empirically from detailed clinical history provided by the parents/carers and patients.

Results 93 (17\%) out of 543 children reviewed had ADHD. Prevalence of sleep difficulties was higher among the ADHD children (52\%) compared to $30 \%$ in the whole cohort. A significantly higher proportion of ADHD patients with sleep problems were on treatment with Melatonin compared to those without ADHD (75\% vs 56\%).

There was a statistically significant association between the degree of socioeconomic deprivation and the prevalence of ADHD and or sleep difficulties. The proportion of children with either or both ADHD and sleep difficulties living in the most deprived (Quintile 1) areas was four times higher than those from the most affluent areas (11.2\% vs $2.8 \%)$.

Conclusion Identification and effective management of daytime sleepiness, sleep-disordered breathing or problematic behaviours at bedtime and upon awakening at night can profoundly improve the symptoms of ADHD in children and adolescents. Evidence-based guidelines for the management of sleep problems in children with ADHD are urgently needed.

\section{G468 ADHD MANAGEMENT - A QUALITY IMPROVEMENT PROJECT}

B Khan, G Rajendran, N Sylvester, K Ganjam, P Mani. Community Paediatrics, Mid Yorkshire Hospitals NHS Trust, Wakefield, UK

10.1136/archdischild-2018-rcpch.456

Attention Deficit Hyperactivity Disorder has an incidence of $3 \%-5 \%$ among school-going children; $60 \%$ of which will continue to have symptoms into adulthood. The aim of this project was to assess all aspects of service delivery to these children as per NICE guidelines at Mid Yorkshire Hospitals NHS Trust.

Methods The first cycle was run retrospectively in September -November 2016. An audit tool was used that reviewed children attending clinics in August 2016. Patients with a diagnosis after 1 st January 2011 were included. The patients were reviewed for details at time of diagnosis or management.

Following the audit, a form was developed to ensure that essential information was documented properly. The unit liaised with local services to improve parent and children workshops availability. A re-audit was run for patients attending in August 2017 using the same parameters to complete the audit loop.

Results 74 patients were included in 2016, while 50 patients have been studied in 2017 (table 1).

\begin{tabular}{lll} 
Abstract G468 Table 1 & & \\
\hline Details at diagnosis & $\mathbf{2 0 1 6}$ & $\mathbf{2 0 1 7}$ \\
\hline Developmental history & $90 \%$ & $100 \%$ \\
Mental state assessment & $74 \%$ & $100 \%$ \\
Observer report used & $100 \%$ & $100 \%$ \\
Abnormal score in observer report & $96 \%$ & $100 \%$ \\
Special education needs noted & $27 \%$ & $59 \%$ \\
Parental training offered & $50 \%$ & $78 \%$ \\
Child social skills training offered & $31 \%$ & $82 \%$ \\
Drug treatment & $100 \%$ & $63 \%$ \\
Baseline weight and height plotted & $100 \%$ & $100 \%$ \\
Baseline blood pressure noted & $63 \%$ & $92 \%$ \\
Baseline heart rate noted & $17 \%$ & $33 \%$ \\
Cardiovascular examination documented & $53 \%$ & $52 \%$ \\
Blood pressure noted at review & $10 \%$ & $72 \%$ \\
Treatment of co-existing conditions at & $100 \%$ & $100 \%$ \\
review & & \\
Side effects at review & $100 \%$ & $100 \%$ \\
\hline
\end{tabular}

Conclusion The project reveals improvement in compliance with NICE guidelines, particularly for monitoring of patients $\mathrm{BP}$ on drug treatment, as well as better education for parents and children. However, parental training and child social skills training are not available to all families due to limited resources.

\section{G469 IMPACT OF CAPSS AND BPSU IN IDENTIFYING THE IMMINENT NEEDS OF ADHD PATIENTS IN TRANSITION FROM CHILDRENS TO ADULTS SERVICES ACROSS THE UNITED KINGDOM}

1,2,3 H Ayyash, ${ }^{2,4} \mathrm{TJ}$ Ford, ${ }^{4} \mathrm{~A}$ Janssens, ${ }^{4} \mathrm{H}$ Eke, ${ }^{4} \mathrm{~A}$ Price, ${ }^{3} \mathrm{M}$ Morton, ${ }^{2,3} \mathrm{R}$ Lynn. ${ }^{1}$ Integrated Neurodevelopmental Children's Services, Cambridgeshire and Peterborough NHS Trust, Peterborough, UK; ${ }^{2}$ Child and Adolescent Surveillance System (CAPSS), RCPsychiatry, London, UK; ${ }^{3}$ British Paediatric Surveillance Unit, RCPCH, London, UK; ${ }^{4}$ Child Mental Health Group, Institute of Health Research, University of Exeter Medical School, Exeter, UK

\subsection{6/archdischild-2018-rcpch.457}

Background The Child and adolescent Psychiatry Surveillance System(CAPSS) is the British Paediatric Surveillance Unit's (BPSU's) baby sibling. It was established in year 2009, and to date has completed more than 6 National studies on children's mental health, with one more in the field of trnsition in ADHD.

Aim 1 st: To dissminate the clinically important findings from the National Transitional ADHD study run on the CAPSS and the BPSU in the UK.

2nd: To raise awareness of CAPSS methodology and its relevance for clinical practice for clinicians and for researchers working with people across the life span.

Methods This ADHD surveillance study run in collaboration with the Child and Adolescent Psychiatric Surveillance System (CAPSS) and British Paediatric Surveillance Unit(BPSU).Each month these units mailed a tick box response card to all consultant paediatricians and child psychiatrists in the UK. Consultants reported on the number of young patients who needed ongoing medications for ADHD and is within six 